\title{
Crosstalk between COVID-19 and prostate cancer
}

\author{
Hisham F. Bahmad (10 ${ }^{1,2} \cdot$ Wassim Abou-Kheir $\mathbb{B}^{1}$
}

Received: 25 May 2020 / Revised: 9 July 2020 / Accepted: 17 July 2020 / Published online: 24 July 2020

C) Springer Nature Limited 2020

\begin{abstract}
A new coronavirus, named SARS-CoV-2, emerged in Wuhan city, China, in December 2019 causing atypical pneumonia and affecting multiple body organs. The rapidly increasing numbers of infected patients and deaths due to COVID-19 disease necessitated declaring it as a global pandemic. Efforts were combined since then to rapidly develop a treatment and/ or a vaccine to combat the deadly virus. Drug repurposing approach has been pursued as a temporary management tactic to treat COVID-19 patients. However, reports about the efficacy of many of the used drugs had been controversial with a dire need to keep the ongoing efforts for rapid development of new treatments. Promising data came out pointing to a possible hidden liaison between prostate cancer (PCa) and COVID-19, where androgen-deprivation therapies (ADT) used in PCa had been shown to instigate a protective role against COVID-19. Delving into the possible mechanisms underlying the crosstalk between COVID-19 and PCa alludes a potential association between SARS-CoV-2 targets on host epithelial cells and PCa genetic aberrations and molecular signatures, including AR and TMPRSS2. The question remains: Can PCa treatments serve as potential therapeutic options for COVID-19 patients?
\end{abstract}

\section{Perspective}

In December 2019, a new coronavirus, named severe acute respiratory syndrome coronavirus-2 (SARS-CoV-2), emerged in Wuhan city, Hubei Province, in China causing severe acute respiratory distress syndrome and atypical pneumonia outbreak. Due to the increasing number of infected patients across the globe, the World Health Organization (WHO) made the assessment that COVID-19 can be characterized as a pandemic on March 11, 2020, and announced a state of global health emergency to combine efforts to contain the spread of the virus and rapidly develop a treatment and/or a vaccine for it. As of mid-July 2020, the virus has infected more than 14 million people around the world, killing more than 600,000 and affecting 216 countries globally [1]. Drug repurposing approach has been pursued as a temporary management tactic to treat

Wassim Abou-Kheir

wa12@aub.edu.lb

1 Department of Anatomy, Cell Biology, and Physiological Sciences, Faculty of Medicine, American University of Beirut, Beirut, Lebanon

2 Arkadi M. Rywlin M.D. Department of Pathology and Laboratory Medicine, Mount Sinai Medical Center, Miami Beach, FL, USA
COVID-19 patients. However, the efficacy of many of the clinically approved drugs tried had been controversial. Therefore, it is still a global priority to keep the efforts ongoing for rapid development of new treatments against SARS-CoV-2.

Recently, studies have been coming out pointing to a possible hidden liaison between prostate cancer $(\mathrm{PCa})$ and COVID-19. Indeed, a study revealed that $\mathrm{PCa}$ patients receiving androgen-deprivation therapy (ADT) had a significantly lower risk (fourfold) of SARS-CoV-2 infection compared with patients not receiving ADT or even patients with any other cancer type [2]. Delving deep into the possible mechanisms underlying the protective role of $\mathrm{PCa}$ therapies against COVID-19 reveals a potential association between SARS-CoV-2 targets on host epithelial cells on one hand, and PCa genetic aberrations and molecular signatures, such as androgen receptor $(A R)$ and transmembrane protease, serine 2 (TMPRSS2), on the other hand [3] (Fig. 1). So far, two main genes have been associated with entry of the COVID-19 virus into host alveolar epithelial cells, namely angiotensin-converting enzyme 2 (ACE2) and TMPRSS2, via binding of spike (S) glycoprotein of coronaviruses to specific cellular ACE2 receptors and its subsequent cleavage (priming) by cellular TMPRSS2 proteases in TMPRSS $2+$ cells or cysteine proteases cathepsin $\mathrm{B}$ or $\mathrm{L}$ (cathepsin $\mathrm{B} / \mathrm{L}$ ) in TMPRSS2 - cells [4]. 


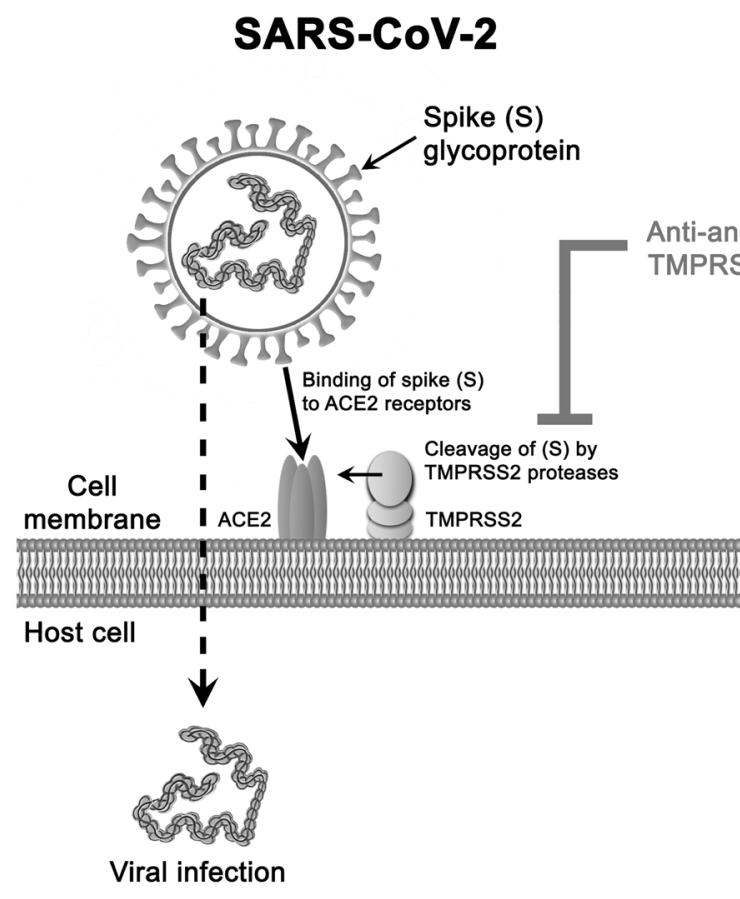

Fig. 1 Schematic representation of the crosstalk between COVID19 and prostate cancer. Potential association is present between SARS-CoV-2 targets on host epithelial cells on one hand, and prostate cancer genetic aberrations and molecular signatures, such as $A R$ and

\section{Prostate Cancer}

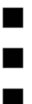

rogen drugs

drogen drugs
S2 inhibitors

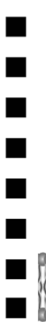

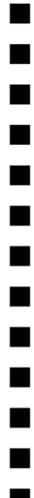

.

TMPRSS2, on the other hand. Antiandrogen drugs and TMPRSS2 inhibitors used in prostate cancer might hence serve as common therapeutic options for COVID-19 patients.
Reports have lately emerged contemplating the possible role of AR sensitivity in increasing susceptibility of patients to SARS-CoV-2 infection via regulating TMPRSS2 transcription [5], which is in turn crucial for SARS-CoV-2 entry into its target cells and initiation of the infectious process [3]. The central role of TMPRSS2 in provoking viral entry into the host lung cells also extends to other respiratory viruses wherein infecting Tmprss $2-/-$ mice with H1N1 influenza virus failed to cause serious infection and protected them from lung disease compared with wild-type mice [6]. In COVID-19, it has been hypothesized that higher androgen levels and hence sustained TMPRSS2 expression among males might explain their predominance in numbers of deaths from the disease versus females $[2,3]$. Another key player in the pathogenesis of COVID-19 is $A C E 2$, which is expressed on epithelial cells of the lungs and is used as a cell receptor for SARS-CoV-2 entrance into the host cell [7]. Interestingly, this gene is also highly expressed in the male urogenital system organs, including the prostate, and indeed it has been postulated that patients with chronic urinary diseases might be more susceptible for SARS-CoV-2 infection than others [8].

In the prostate gland, upon activation of AR by androgen binding, it undergoes conformational change instigating transcription of target genes, such as prostate-specific antigen and TMPRSS2. The latter androgen-regulated TMPRSS2 gene fuses to ERG driving PCa initiation and progression in almost $50-70 \%$ of $\mathrm{PCa}$ patients [9]. Therefore, in androgen-sensitive $\mathrm{PCa}$, the standard first-line therapy is still ADT ever since 1941 until today. In addition to ADT, an alternative approach to directly target and modulate TMPRSS2 expression is via protease inhibitors that impair the activity of TMPRSS2, such as Camostat, Nafamostat, and Bromhexine [10]. Many of those drugs are currently under investigation in clinical trials on COVID-19 patients. Moreover, we hypothesize that any drug which downregulates TMPRSS2 expression through targeting AR, AR co-regulatory factors, or AR downstream transcription 
factors might be potentially effective against COVID-19 and is worth investigating under a clinical trial. Noteworthy mentioning that a phase III clinical trial will be started soon to assess the efficacy of 13-cis-retinoic acid (isotretinoin) a retinoid used in severe acne due to hyperandrogenismin the treatment of COVID-19 (ClinicalTrials.gov; NCT04353180; estimated study start date June 2020).

Collectively, these pieces of evidence suggest that a crosstalk indeed exists between COVID-19 and PCa at a subcellular genetic level. The high expression of TMPRSS 2 and its role in the pathogenesis of both diseases paves the way for identifying novel therapeutic approaches to treat COVID-19 that are based on androgen suppression and TMPRSS2 protease inhibition. What is really promising about PCa treatments being repurposed to target SARS$\mathrm{CoV}-2$ is that many of those drugs have low risk of serious side effects and thus can be used solely or in combination with other potential repositioned drugs to study their efficacy against COVID-19.

Author contributions HFB and WAK conceived the concept and idea of the present work. HFB drafted the paper. WAK and HFB critically revised the paper and approved the final draft.

\section{Compliance with ethical standards}

Conflict of interest The authors declare that the research was conducted in the absence of any commercial or financial relationships that could be construed as a potential conflict of interest.

Publisher's note Springer Nature remains neutral with regard to jurisdictional claims in published maps and institutional affiliations.

\section{References}

1. World Health Organization. Coronavirus disease (COVID-19) pandemic. 2020.

2. Montopoli M, Zumerle S, Vettor R, Rugge M, Zorzi M, Catapano $\mathrm{CV}$ et al. Androgen-deprivation therapies for prostate cancer and risk of infection by SARS-CoV-2: a population-based study $(n=$ 4532). Ann Oncol. 2020. Epub ahead of print.

3. Stopsack KH, Mucci LA, Antonarakis ES, Nelson PS, Kantoff PW. TMPRSS2 and COVID-19: serendipity or opportunity for intervention? Cancer Discov. 2020:1-6. https://doi.org/10.1158/ 2159-8290.CD-20-0451.

4. Hoffmann M, Kleine-Weber H, Schroeder S, Krüger N, Herrler T, Erichsen S, et al. SARS-CoV-2 cell entry depends on ACE2 and TMPRSS2 and is blocked by a clinically proven protease inhibitor. Cell. 2020;181:271-80.e278.

5. Wambier CG, Goren A, Vaño-Galván S, Ramos PM, Ossimetha A, Nau $\mathrm{G}$ et al. Androgen sensitivity gateway to COVID-19 disease severity. Drug Dev Rese. 2020.

6. Hatesuer B, Bertram S, Mehnert N, Bahgat MM, Nelson PS, Pöhlmann S, et al. Tmprss2 is essential for influenza H1N1 virus pathogenesis in mice. PLoS Pathog. 2013;9:e1003774.

7. Li M-Y, Li L, Zhang Y, Wang X-S. Expression of the SARS$\mathrm{CoV}-2$ cell receptor gene ACE2 in a wide variety of human tissues. Infect Dis Poverty. 2020;9:45.

8. Wu Z-s, Zhang Z-q, Wu S. Focus on the crosstalk between COVID-19 and urogenital systems. J Urol. 2020. https://doi.org/ 10.1097/JU.0000000000001068.

9. Pettersson A, Graff RE, Bauer SR, Pitt MJ, Lis RT, Stack EC, et al. The TMPRSS2:ERG rearrangement, ERG expression, and prostate cancer outcomes: a cohort study and meta-analysis. Cancer Epidemiol Biomark Prev. 2012;21:1497-509.

10. Lucas JM, Heinlein C, Kim T, Hernandez SA, Malik MS, True $\mathrm{LD}$, et al. The androgen-regulated protease TMPRSS2 activates a proteolytic cascade involving components of the tumor microenvironment and promotes prostate cancer metastasis. Cancer Discov. 2014;4:1310-25. 